\title{
Solitons and fractional statistics
}

\author{
Alexios P. Polychronakos ${ }^{\text {a* }}$ \\ aTheoretical Physics Dept., Uppsala University \\ Box 803, S-75108 Uppsala, Sweden
}

Solitons in the continuum limit of the Calogero model are derived and shown to correspond to one-particle excitations. The statistical mechanics of exclusion statistics particles is then formulated in terms of a priori probabilities and a path integral is thereoff constructed.

Talk delivered at the Trieste 10-12 April 1995 Conference on statistical mechanics and QFT, and at the Oslo 21-26 August 1995 Worskhop on low-dimensional systems.

\section{INTRODUCTION}

This talk consists, in fact, of two short stories, both connected to or motivated from the Calogero model. In the first, I derive an analytic expression for a solitonic wave excitation in the continuum limit of the Calogero model, and show that it corresponds to one-particle excitations in the quantum description. Large-amplitude waves are also derived and correspond to a two-band quantum state. A conjecture for the chiral hamiltonian of this problem is made. In the second, I formulate exclusion statistics in terms of microscopic occupation probabilities. This leads to a path-integral construction of the partition function for such systems and can be used as a starting point for further generalizations. A bonus of this formalism is that the coefficients of the lowtemperature expansion of the specific heat can be easily evaluated. Much of the material in this talk is taken from references [1].

\section{SOLITONS}

The Calogero-Sutherland-Moser model is exactly solvable in both the classical and the quantum regime 24. This model is related to quantum spin chains with long range interactions between the spins [5], wave propagation in stratified fluids [6], random matrix theory [3,7] and fractional statistics 8]. Remarkably, the quan-

\footnotetext{
${ }^{*}$ Part of this work was done while still at CERN
}

tum solution is much easier to interpret, exhibiting a straightforward analogy to the free fermion case. In a recent paper, Sutherland and Campbell examined the classical system in the thermodynamic limit and identified the excitations [9]. It was found that the classical system has solitons, corresponding to a single particle running through the rest of them, as well as small amplitude waves (phonons), identified with holes. I shall derive here large amplitude wave and soliton solutions of the classical system in the continuous limit, where the particles form a "fluid," and examine their correspondence to the quantum states.

Consider a collection of particles of unit mass with the hamiltonian

$H=\frac{1}{2} \sum_{i=1}^{N} \dot{x}_{i}^{2}+\sum_{i>j} \frac{g}{\left(x_{i}-x_{j}\right)^{2}}$

We shall be interested in the limit $N, L \rightarrow \infty$ with $N / L$ fixed. In this limit, the system can be described in terms of a density field $\rho(x)$ and a velocity field $v(x)$. At equilibrium, the particles will form a regular lattice of spacing $a$ and density $\rho_{o}=1 / a$. The particle current is $J=\rho v$ and by particle conservation

$\dot{\rho}+\partial J=\dot{\rho}+\partial(\rho v)=0$

where $\partial=\partial / \partial x$. The kinetic energy of the system is

$K=\int d x \frac{1}{2} \rho v^{2}$ 
We can formally solve eq. (2) for $v$ to obtain $v=-\partial^{-1} \dot{\rho} / \rho$, and the expression for the kinetic energy becomes

$K=\int d x \frac{\left(\partial^{-1} \dot{\rho}\right)^{2}}{2 \rho}$

This is exactly the kinetic term of the collective field hamiltonian description of a many-body system 10]. The potential energy can also be expressed in terms of the density. The naive expression, however is incorrect, the reason being that the interaction is singular at coincidence points, and thus a substantial part of the potential energy comes from nearest neighbors. The correct expression requires a careful conversion of the discrete sum in terms of the continuous fields. Alternatively, we can simply take the classical limit $(\hbar \rightarrow 0)$ of the quantum mechanical expression derived in the collective field formulation [11. The result is

$V=\int d x\left\{\frac{\pi^{2} g}{6} \rho^{3}-\frac{g}{2} \rho \partial \tilde{\rho}+\frac{g}{8} \frac{(\partial \rho)^{2}}{\rho}\right\}$

where $\tilde{\rho}$ stands for the Hilbert transform:

$\tilde{\rho}=\int d y P \cdot P \cdot \frac{1}{x-y} \rho(y)$

The dynamics of the system can be found by varying the lagrangian $L=K-V+\mu \rho$ with respect to $\rho$. The chemical potential $\mu$ plays the role of a Lagrange multiplier ensuring that the total number of particles remains constant. The resulting equations of motion are

$\partial^{-1} \dot{v}+\frac{1}{2} v^{2}+\frac{\pi^{2} g}{2} \rho^{2}-g \partial \tilde{\rho}+\frac{g}{8}\left(\frac{\partial \rho}{\rho}\right)^{2}-g \frac{\partial^{2} \rho}{4 \rho}=\mu(7)$

as well as the continuity equation. By requiring that the static configuration $v=0, \rho=\rho_{o}$ be a solution, we obtain the value of the chemical potential

$\mu=\frac{\pi^{2} g}{2} \rho_{o}^{2}$

For a localized constant profile configuration, propagating at speed $\mathrm{v}$, both $\rho$ and $v$ are functions of $x-\mathrm{v} t$ only. From the continuity equation we have

$\partial(v \rho-\mathrm{v} \rho)=0 \quad$ and thus $\quad v=\frac{\rho-\rho_{o}}{\rho} \mathrm{v}$
In the above, the integration constant is fixed by the boundary condition that $v \rightarrow 0$ at $x \rightarrow \pm \infty$, where $\rho \rightarrow \rho_{o}$. Similarly, the eq. of motion becomes

$\frac{\mathrm{v}^{2}}{2 g}\left(\frac{\rho_{o}^{2}}{\rho^{2}}-1\right)+\frac{\pi^{2}}{2}\left(\rho^{2}-\rho_{o}^{2}\right)=\partial \tilde{\rho}-\frac{1}{8}\left(\frac{\partial \rho}{\rho}\right)^{2}+\frac{\partial^{2} \rho}{4 \rho}(10)$

A solution of the above equation is

$\rho_{\text {sol }}=\rho_{o}\left(1+\frac{u}{\left(\pi \rho_{o} x\right)^{2}+u^{2}}\right), \quad u=\frac{\mathrm{v}_{s}^{2}}{\mathrm{v}^{2}-\mathrm{v}_{s}^{2}}(11)$

provided that $\mathrm{v}>\mathrm{v}_{s}$.

The above soliton carries particle number $Q$, momentum $P$ and energy $E$, defined as the extra amount over the static solution $\rho_{o}$. We find

$Q=\int d x\left(\rho_{\mathrm{sol}}-\rho_{o}\right)=1$

$P=\int d x \rho_{\text {sol }} v=\mathrm{v}$

$E=\int d x\left[K\left(\rho_{\text {sol }}\right)+V\left(\rho_{\text {sol }}\right)-V\left(\rho_{o}\right)\right]=\frac{1}{2} \mathrm{v}^{2}$

We observe that the net particle number carried by the soliton is 1 , independently of its velocity; its momentum and energy are also those of a free particle of unit mass moving at the soliton velocity v. Therefore, the soliton can be exactly identified with a particle excitation of the system. This is in agreement with exact results drawn from the quantum theory, where particle excitations always move faster than the sound [9] The form of the above soliton, however, is at odds with the results found in [9]. We suspect that the source of the discrepancy is the truncation to a finite number of $x$-derivatives of the form for the potential in [9]; this turns the equation to a local one and gives the soliton an exponential decay, rather than the inverse-square decay of the nonlocal equation.

A finite-amplitude periodic solution for the equations of motion is

$\rho_{\text {wave }}=\rho_{o}+\frac{1}{\lambda}\left(\frac{1}{\sqrt{\lambda^{2} A^{2}+1}-\lambda A \cos \frac{2 \pi x}{\lambda}}-1\right)$

where

$\mathrm{v}=\left(\mathrm{v}_{s}-\frac{\pi \sqrt{g}}{\lambda}\right) \sqrt{1+\frac{2 A^{2}\left(\lambda \rho_{o}-1\right)}{\rho_{o}^{2}\left(1+\sqrt{\lambda^{2} A^{2}+1}\right)}}$ 
$A$ is the amplitude of the wave and $l$ its wavelength. The above equation is, therefore, the amplitude-dependent dispersion relation in this nonlinear system. Note that in the limit $\lambda \rightarrow \infty$ the above equations reduce to the single soliton solution.

In summary, we have found exact soliton and wave solutions for the CS system in the continuum limit. Certainly the above do not exhaust the list of solutions; the general motion of the system will be a nonlinear superposition of waves (or solitons). It is instructive to put the above solutions into correspondence with the quantum mechanical states. Consider $N$ particles in a space of length $L$. The ground state of the system consists of a "Luttinger sea" in the pseudomomentum, with spacing between adjacent particles equal to $2 \pi \ell / L$ and "Fermi level" $\pi \ell N / L$, where $g=\ell(\ell-\hbar)$. At the limit $\hbar \rightarrow 0, N, L \rightarrow \infty$, $N / L \rightarrow \rho_{o}$, the ground state becomes a continuous filled band with Fermi level $P_{F}=\pi \sqrt{g} \rho_{o}$. A small amplitude wave, corresponding to a hole, is a very small gap in the band. A soliton, corresponding to a particle excitation, is a single particle peeled from the Fermi level and placed some distance above. The generic finite amplitude wave corresponds to a state with two continuous filled bands, of widths $P_{1}$ and $P_{2}$ (with $P_{1}+P_{2}=2 P_{F}$ ) and with a gap $G$ between them. These are related to the wave parameters as

$\lambda=\frac{2 \pi \sqrt{g}}{P_{1}}$

$\mathrm{v}=\frac{P_{2}}{2}\left(\frac{G}{\pi \sqrt{g} \rho_{o}}+1\right)$

Such a state can be visualized as arising either by successively exciting single particles by the same constant momentum, until they form a continuous band, or by gradually augmenting the gap of a hole, until it becomes finite. This state can thus be thought of as either a coherent state of solitons, or as a coherent state of phonons, their nonlinear nature accounting for the change in profile as they accumulate. Indeed, the soliton itself can be thought of as a superposition of many phonons with very large wavenumber, and the phonon as a superposition of many solitons just above the
Fermi level. For the finite $N$ (finite $L$ ) system the distinction between the two is fuzzy and in principle only one kind of excitations need be considered as fundamental. Note, further, that quantum mechanically the holes behave as particles with fractional statistics of order $\hbar / \ell$ (meaning that $\ell / \hbar$ of them put together would form a fermion). At the classical limit $\hbar \rightarrow 0$, thus, they become bosons, as they should be since phonons obey no exclusion principle. Particles, on the other hand, carry statistics of order $\ell / \hbar$. Thus in the classical limit they become "superfermions" meaning that no two of them can occupy relatively nearby quantum states. This is consistent with the inverse square repulsion between the classical particles. It should be noted that a similar soliton solution exists in the fluid description of this problem keeping the $\hbar$ corrections [14], and it would be intersting to find its interpretation.

We conclude by noting that the quantum mechanical problem separates into two noninteracting chiral sectors, having to do with excitations near either end of the Luttinger sea. (The two sectors mix nonperturbatively when a number of particles of order $N$ is excited, depleting the sea.) Therefore, the equation governing the continuum system should also decompose into two nonmixing, first-order in time equations, one for each sector. For the corresponding equation for free fermions this is indeed the case [12]. In fact, from the collective field description of the system when only one chiral sector is present, we deduce that the chiral equations are exactly of the BenjaminOno type 11,13. The exact field combinations in terms of which this decomposition would be achieved, however, are not known and constitute an open problem.

\section{EXCLUSION STATISTICS}

Statistics is an inherently quantum mechanical property of identical particles which, as the name suggests, modifies the statistical mechanical properties of large collections of such particles. Motivated by properties of the Haldane-Shastry model (a lattice version of the Calogero model), Haldane defined a generalized exclusion statistics through the reduction of the Hilbert space of ad- 
ditional particles in a system due to the ones already present in the system [15. He proposed then the definition

$g=-\frac{\Delta d}{\Delta N}$

where $N$ is the number of particles in the system, $d$ is the dimensionality of the single-particle Hilbert space, obtained by holding the quantum numbers of $N-1$ particles fixed, and $\Delta d$ and $\Delta N$ are their variation keeping the size and boundary conditions of the system fixed. $g=0$ corresponds to bosons (no exclusion) while $g=1$ corresponds to fermions, excluding a single state for the remaining particles, the one they occupy.

On the basis of the above, Haldane proposed the combinatorial formula for the number of many-body states of $N$ particles occupying a group of $K$ states

$M=\frac{[K-(g-1)(N-1)] !}{N ![K-g(N-1)-1] !}$

Starting from this, the thermodynamical properties of particles with exclusion statistics can be derived 16 18], and this system has received a lot of recent attention 1922 .

It is obvious from that exclusions statistics makes sense only in a statistical sence, since $\Delta d$ and $M$ can become fractional for $\Delta N=1$ or $N=1$. It is, nevertheless, useful to attempt a microscopic realization and interpretation of fractional exclusion statistics, and see what it implies for the one-particle states. Such a description has the obvious advantage of being generalizable to interacting particles, for which the notion of $d$ becomes hard to define.

The starting point will be the grand partition function for exclusion-statistics particles in $K$ states

$Z(K)=\sum_{N=0}^{\infty} M(K, N) x^{N}$

where we put $x=\exp (\mu-\varepsilon) / k T$ with $\mu$ the chemical potential and assumed that all $K$ states are at the same energy $\varepsilon$. In the statistical limit of large $K, Z(K)$ should be extensive. This introduces, then, the notion of a microscopic description of the system in which the above $Z$ is the
$K$-th power of a single-state partition function. Each single level can be occupied by any number of particles, but with an a priori probability $P_{n}$ for each occupancy $n$ independent of the temperature. We thus demand

$Z(K, x)=\left(\sum_{n} P_{n}(K) x^{n}\right)^{K}=\sum_{N=0}^{\infty} M(K, N) x^{N}$

for all $x$. The above probabilities must, in general, depend on $K$. If, however, $P_{n}(K)$ assume some (finite) asymptotic values as $K$ goes to infinity (as they should for an extensive $Z$ ), then the above microscopic partition function becomes an accurate description in the statistical limit. This indeed happens, and we obtain

$P_{n}=\prod_{m=2}^{n}\left(1-\frac{g n}{m}\right)$

The most obvious feature of the above expressions is that, unless $g=0,1$, they always become negative for some values of $n$. Therefore, their interpretation as probabilities is problematic. This is an inherent problem of fractional $g$-statistics which cannot be rectified by, e.g., truncating $M(K, N)$ to zero for $N>K / g$. The description of the statistical system in terms of effective negative microscopic probabilities is, nevertheless, accurate and useful. Note, also, that the above $P_{n}$ never truncate to zero for $n$ above some maximal value (unless $g=1$ ), unlike parafermions.

From the above, the single-level partition function $Z(x) \equiv Z$ can be shown to satisfy

$Z^{g}-Z^{g-1}=x$

The average occupation number $\bar{n}$ is expressed as

$\bar{n}=\frac{1}{Z} x \partial_{x} Z=x \partial_{x} W$

where $W=\ln Z$ is the thermodynamic potential $($ over $-k T)$. It can be shown that the above imply

$(1-g \bar{n})^{g}[1-(g-1) \bar{n}]^{1-g}=\bar{n} x^{-1}$

in accordance with the result of [16 18].

From the above we can easily calculate the lowtemperature expansion coefficients of the energy 
(or specific heat) in terms of the temperature. Except for factors depending on the dimensionality of space, these are [21]

$C_{n}=\int_{0}^{1} \frac{d x}{x}(\ln x)^{n}\left[(-1)^{n} \bar{n}(x)+\bar{n}\left(x^{-1}\right)-\frac{1}{g}\right](27)$

where $1 / g$ is the saturation density for $n$ at zero temperature and $\varepsilon<\mu$. The trick is to use (25) and change variable of integration from $x$ to $W$. Since by (24) $x$ is an explicit expression of $W$, we obtain the explicit integral

$$
\begin{array}{r}
C_{n}=(-1)^{n} \int_{0}^{\infty}\left\{\left[g W+\ln \left(1-e^{-W}\right)\right]^{n}\right. \\
\left.-(g W)^{n}\right\} d W
\end{array}
$$

The first few coefficients are

$C_{0}=0, \quad C_{1}=\frac{\pi^{2}}{6}, C_{2}=-\zeta(3)(1+g)$, etc.

We see that $C_{0}=0$ for all $g$, as conjectured in [21]. This expresses the fact that the ground state of the many-body $g$-on system is nondegenerate, which is expected to be a generic feature of particle systems. $C_{1}$ is also independent of $g$. This result has first been conjectured by S. Isakov and then numerically verified by J. Myrheim. The first analytical derivation was provided by D. Arovas [23]. In fact, from (28) we see that $C_{n}$ is a polynomial in $g$ of degree $n-1$, its $k$-th coefficient being

$C_{n, k}=(-1)^{n}\left(\begin{array}{l}n \\ k\end{array}\right) \int_{0}^{\infty} W^{k}\left[\ln \left(1-e^{-W}\right)\right]^{n-k} d W$

Through a change of variables we can show that the above coefficients obey

$C_{n, k}=C_{n, n-k-1}$

so only half of the coefficients need be calculated.

The above relation points to a mapping between the small- $g$ and large- $g$ regions. Indeed, this is related to a duality relation in these systems. It is easy to see that $Z$ satisfies:

$Z^{-1}\left(g, x^{-g}\right)+Z^{-1}\left(g^{-1}, x\right)=1$

From the above relation, the duality relation for the density is recovered 18,21

$g \bar{n}(g, x)+g^{-1} \bar{n}\left(g^{-1}, x^{-1 / g}\right)=1$
We regard the formula for $Z(32)$ as more fundamental since it seems to be more generic. For instance, parafermions of order $p=1 / g$ are defined such that at most $p$ particles can be put per state with probabilities 1 . Thus

$Z_{\text {par }}=1+x+\cdots x^{p}=\frac{1-x^{p+1}}{1-x}$

from which we can write the generalized parafermionic partition function $Z_{p a r}(g, x)$ by simply putting $p=1 / g$ above. It can be seen that $Z_{\text {par }}$ also satisfies (32) but not (33).

The free energy $W$ can be expressed as a power series in $x$

$W=\sum_{n=1}^{\infty} \frac{w_{n}}{n} x^{n}$

in terms of the "connected" weights

$w_{1}=P 1, w_{2}=2 P_{2}-P_{1}^{2}, w_{3}=3 P_{3}-3 P_{1} P_{2}+P_{1}^{3}$

etc. We find for $w_{n}$ :

$w_{n}=\prod_{m=1}^{n-1}\left(1-\frac{g n}{m}\right)$

These are remarkably similar to $P_{n}$ (except for the range of $m$ ). Notice that the $w_{n}$ are not probabilities, but rather cluster coefficients. In fact, $w_{n}=1$ for bosons and $w_{n}=(-)^{n-1}$ for fermions. Also, $w_{2}=1-2 g$ [19].

From the above expressions for $w_{n}$ we can find a path integral representation for the partition function of exclusion particles in an arbitrary external potential. We start from the usual euclidean path integral with periodic time $\beta$ for $N$ particles with action the sum of $N$ one-particle actions, and sum over all particle numbers $N$ with appropriate chemical potential weights. Since the particles are identical, we must also sum over paths where particles have exchanged final positions, with weights equal to the inverse symmetry factors of the permutation to avoid overcounting (compare with Feynman diagrams). Thus the path integral for each $N$ decomposes into sectors labeled by the elements of the permutation group $\operatorname{Perm}(N)$. By the usual argument, the free energy will be given by the sum of all connected 
path integrals. It is obvious that these are the ones where the final positions of the particles are a cyclic permutation of the original ones (since these are the only elements of Perm $(N)$ that cannot be written as a product of commuting elements). These have a symmetry factor of $1 / N$ corresponding to cyclic relabelings of particle coordinates (compare with the factors of $1 / n$ included in (35)). They really correspond to one particle wrapping $N$ times around euclidean time. Thus, if we weight these configurations with the extra factors $w_{N}$, as we have the right to do since they belong to topologically distinct sectors, we will reproduce the free energy of a distribution of $g$-statistics particles on the energy levels of the one-body problem, that is

$\mathcal{W}(\beta, \mu)=\sum_{N=1}^{\infty} e^{\mu N} \int \frac{w_{N}}{N} \prod_{n=1}^{N} D x_{n}\left(t_{n}\right) e^{-S_{E}\left[x_{n}\left(t_{n}\right)\right]}$

where $S_{E}$ is the one-particle euclidean action and the paths obey the boundary conditions $x_{n}(\beta)=$ $x_{n+1}(0), x_{N}(\beta)=x_{0}(0)$. $\quad(x$ can be in arbitrary dimensions.) The partition function will be the path integral over all (connected and disconnected) paths, with appropriate symmetry factors and a factor of $w_{n}$ for each connected $n$-particle component.

It is clear that the above path integral is not unitary, since the weights $w_{n}$ are not phases, nor does it respect cluster decomposition, since the $w_{n}$ do not provide true representations of the permutation group (unlike the $g=0,1$ cases). This is again a manifestation of the non-microscopic nature of exclusion statistics. It does make sense, nevertheless, at the statistical limit.

The above path-integral realization can be extended to other statistics. E.g., for parafermions of order $p$ (with $p$ integer) the corresponding weigts $w_{n}$ are

$w_{n}=-p$ for $n=0 \bmod (p+1), 1$ otherwise.(39)

This representation is more economical than the one calling for $p$ distinct flavors of fermions and projecting into invariant states (singlets) under $\operatorname{Perm}(p)$. The origin of the apparent nonunitarity and breakdown of cluster decomposition in the above integral for parafermions is clear: it is due to the above projection, which must be inserted in the (unitary) many-flavor path integral.

The possibility to define statistics through the choice of the coefficients $w_{n}$ suggests other possible generalizations. Perhaps the simplest one is to choose

$w_{n}=(-\alpha)^{n-1}$

that is, one factor of $-\alpha$ for each unavoidable particle crossing. This leads to the statistical distribution for the average occupation number $\bar{n}$

$\bar{n}=\frac{1}{e^{(\varepsilon-\mu) \beta}+\alpha}$

which is the simplest imaginable generalization of the Fermi and Bose distribution, and was extensively analyzed in 24]. The combinatorial for(38) mula for putting $N$ particles in $K$ states for the above $\alpha$-statistics is

$M=\frac{K(K-\alpha)(K-2 \alpha) \cdots(K-(N-1) \alpha)}{N !}$

This can be thought as a different realization of the exclusion statistics idea: the first particle put in the system has $K$ states to choose, the next has $K-\alpha$ due to the presence of the previous one an so on, and dividing by $N$ ! avoids overcounting. Fermions and bosons correspond to $\alpha=1$ and $\alpha=-1$ respectively, while $\alpha=0$ corresponds to Boltzmann statistics (as is also clear from the path integral, in which no configurations where particles have exchanged positions are allowed, but factors of $1 / N$ ! are still included). The corresponding single-level probabilities are

$P_{n}=\prod_{m=1}^{n-1} \frac{1-m \alpha}{1+m}$

For $\alpha=1 / p$ with $p$ integer (a fraction of a fermion), the above probabilities are all positive for $n$ up to $p$ and vanish beyond that. For $\alpha<0$ all probabilities are positive and nonzero. Thus, the above system has a bosonic $(\alpha<0)$ and a fermionic $(\alpha>0)$ sector, with Boltzmann statistics as the separator. It is a plausible alternative definition of exclusion statistics, due to (42), and has many appealing features, not shared by the standard (Haldane) exclusion statistics, such 
as positive probabilities, a maximum single-level occupancy in accordance with the fraction of a fermion that $\alpha$ represents, and analytic expressions for all thermodynamic quantities. It would be interesting to find a physical system in which these statistics are realized.

We conclude by pointing out that, once we have the path integral we can easily extend the notion of exclusion statistics to interacting particles: we simply replace the action $\sum_{n} S_{E}\left[x_{n}\right]$ by the full interacting $N$-particle action, thus circumventing all difficulties with combinatorial formulae. In the interacting case one has to work with the full partition function, rather than the free energy, since topologically disconnected diagrams are still dynamically connected through the interactions and do not factorize. Applications of the above on physical systems would be welcome.

\section{REFERENCES}

1. A.P. Polychronakos, Phys. Rev. Lett. bf 74, 5153 (1995); Uppsala preprint UUITP-03-95, to appear in Phys. Lett. B.

2. F. Calogero, Jour. of Math. Phys. 10, 2191 and 2197 (1969); 12, 419 (1971).

3. B. Sutherland, Phys. Rev. A4, 2019 (1971); Phys. Rev. A5, 1372 (1972); Phys. Rev. Lett. 34, 1083 (1975).

4. J. Moser, "Dynamical Systems, Theory and Applications," Lecture Notes in Physics 38, Springer-Verlag, New York 1975.

5. F. D. M. Haldane, Phys. Rev. Lett. 60, 635 (1988); B. S. Shastry,Phys. Rev. Lett. 60, 639 (1988).

6. H. H. Chen, Y. C. Lee and N. R. Pereira, Phys. Fluids 22 (1979) 187.

7. B. D. Simons, P. A. Lee, and B. L. Altshuler, Phys. Rev. Lett. 70, 4122 (1993);Phys. Rev. Lett. 72, 64 (1994); E. Mucciolo, B. Shastry, B. Simons and B. Altshuler, condmat/9309030.

8. J.M. Leinaas and J. Myrheim, Phys. Rev. B37, 9286 (1988); A. Polychronakos, Nucl. Phys. B234 (1989) 597.

9. B. Sutherland and J. Campbell (preprint), Sept. 1993.

10. N. N. Bogoliubov and D. N. Zubarev, JETP
1 (1955) 83; A. Jevicki and B. Sakita, Nucl. Phys. B165 (1980) 511.

11. I. Andric, A. Jevicki and H. Levine, Nucl. Phys. B215 (1983) 307; I. Andric and V. Bardek, J. Phys. A21 (1988) 2847.

12. J. Polchinski, Nucl. Phys. B362 (1981) 125.

13. J. Minahan and A. P. Polychronakos, Phys. Rev. B50 (1994) 4236.

14. I. Andric, V. Bardek and L. Jonke, Phys. Lett. B357 (1995) 374.

15. F.D.M. Haldane, Phys. Rev. Lett. 67 (1991) 937.

16. S. B. Isakov, Int. Jour. Mod. Phys. A9 (1994) 2563.

17. Y.-S. Wu, Phys. Rev. Lett. 73 (1994) 922.

18. A.K. Rajakopal, Phys. Rev. Lett. 74 (1995) 1048.

19. M.V.N. Murthy and R. Shankar, Phys. Rev. Lett. 72 (1994)

20. A.D. de Veigy and S. Ouvry, Phys. Rev. Lett. 72600.

21. C. Nayak and F. Wilczek, Phys. Rev. Lett. 73 (1994) 2740.

22. D. Karabali and V.P. Nair, Nucl. Phys. B438 (1995) 551.

23. D. Arovas, S. Isakov and J. Myrheim, private communication.

24. R. Acharya and P. Narayana Swamy, J. Phys. A27 (1994) 7247. 